\title{
O desenvolvimento do Transtorno do Espectro Autista está relacionado com a suplementação de altas doses de ácido fólico no período periconcepcional?
}

\author{
Is the development of Autistic Spectrum Disorder related to the supplementation of high doses of \\ folic acid in the periconceptional period? \\ ¿El desarrollo del Trastorno del Espectro Autista está relacionado con la suplementación de altas \\ dosis de ácido fólico en el período periconcepcional?
}

Recebido: 16/11/2021 | Revisado: 24/11/2021 | Aceito: 25/11/2021 | Publicado: 07/12/2021

Isabela Chaves Lira Cruz

ORCID: https://orcid.org/0000-0001-6006-5832 Pontifícia Universidade Católica de Goiás, Brasil

E-mail: is.lira@hotmail.com

Brunna Michelly da Silva Sousa

ORCID: https://orcid.org/0000-0002-3187-3432

Pontifícia Universidade Católica de Goiás, Brasil E-mail: brunna.michelly@outlook.com

Tamyres Borges Pereira

ORCID: https://orcid.org/0000-0002-9721-2506 Pontifícia Universidade Católica de Goiás, Brasil E-mail: tamyresborges94@gmail.com

Irlane Moraes Vasconcelos Souza ORCID: https://orcid.org/0000-0001-7509-3967

Universidade Cidade de São Paulo, Brasil E-mail: Irlanemvs@hotmail.com

Fernanda de Melo Franco Machado ORCID: https://orcid.org/0000-0001-8492-5235

Universidade de Rio Verde, Brasil

E-mail: melofernanda1@hotmail.com

\begin{abstract}
Resumo
O Transtorno do Espectro Autista (TEA) define um grupo de distúrbios do desenvolvimento neurológico, geralmente diagnosticado na primeira infância. Durante as duas últimas décadas foi detectado um aumento crescente da prevalência do TEA. Centenas de estudos foram realizados, no entanto, ainda não foi esclarecido por que a incidência de autismo aumentou rapidamente durante os anos 90 e continua aumentando nos anos 2000. Foi realizado levantamento bibliográfico, nas bases de dados: PUBMED, LILACS e SCIELO, do período entre 2015 a 2020. Foram utilizadas os descritores: "Folic Acid" and "Autism Spectrum Disorder". Inicialmente foram encontrados 46 artigos. Destes, apenas 34 estavam de acordo com os critérios de inclusão e abordavam aspectos relacionados à suplementação de ácido fólico durante o período periconcepcional e sua influência para o desenvolvimento de TEA. Sobre o TEA e a administração de altas doses de ácido fólico durante o período periconcepcional, conclui-se que são necessárias pesquisas adicionais com desenhos de estudos adequadamente padronizados e com metodologia adequada antes que quaisquer conclusões definitivas possam ser tomadas. Os resultados de estudos clínicos recentes, apesar de poucos e inconclusivos, levantam questões sobre dosagens adequadas ou uso mais direcionado do ácido fólico que necessitam ser investigadas.
\end{abstract}

Palavras-chave: Ácido fólico; Transtorno do espectro autista; Autismo.

\begin{abstract}
Autistic Spectrum Disorder (ASD) defines a group of neurodevelopmental disorders, usually diagnosed in early childhood. During the last two decades an increasing increase in the prevalence of ASD has been detected. Hundreds of studies have been carried out, however, it remains unclear why the incidence of autism increased rapidly during the 1990s and continues to increase in the 2000s. A bibliographic survey was carried out in the following databases: PUBMED, LILACS and SCIELO between 2015 and 2020. The following descriptors were used: "Folic Acid" and "Autism Spectrum Disorder". Initially, 46 articles were found. Of these, only 34 met the inclusion criteria and addressed aspects related to folic acid supplementation during the periconceptional period and its influence on the development of ASD. With regard to TEA and the administration of high doses of folic acid during the periconceptional period, it is concluded that further research is needed with appropriately standardized study designs and with adequate methodology before any definitive conclusions can be drawn. The results of recent clinical studies, although few and inconclusive, raise questions about adequate dosages or more targeted use of folic acid that need to be investigated.
\end{abstract}

Keywords: Folic acid; Autistic spectrum disorder; Autism. 


\section{Resumen}

El trastorno del espectro autista (TEA) define un grupo de trastornos del desarrollo neurológico, generalmente diagnosticados en la primera infancia. Durante las últimas dos décadas se ha detectado un aumento creciente en la prevalencia de TEA. Se han realizado cientos de estudios, sin embargo, no está claro por qué la incidencia del autismo aumentó rápidamente durante la década de 1990 y continúa aumentando en la década de 2000. Se realizó una encuesta bibliográfica en las siguientes bases de datos: PUBMED, LILACS y SCIELO entre 2015 y 2020. Se utilizaron los siguientes descriptores: “Ácido fólico” y "Trastorno del espectro autista”. Inicialmente se encontraron 46 artículos. De estos, solo 34 cumplieron con los criterios de inclusión y abordaron aspectos relacionados con la suplementación con ácido fólico durante el período periconcepcional y su influencia en el desarrollo de TEA. Con respecto a la TEA y la administración de altas dosis de ácido fólico durante el período periconcepcional, se concluye que se necesita más investigación con diseños de estudio debidamente estandarizados y con una metodología adecuada antes de poder extraer conclusiones definitivas. Los resultados de estudios clínicos recientes, aunque pocos y no concluyentes, plantean dudas sobre las dosis adecuadas o el uso más específico de ácido fólico que deben investigarse.

Palabras clave: Ácido fólico; Trastorno del espectro autista; Autismo.

\section{Introdução}

O Transtorno do Espectro Autista (TEA) define um grupo de distúrbios do desenvolvimento neurológico que afeta aproximadamente $1 \%$ da população que geralmente é diagnosticado na primeira infância (Guo et al., 2019; Maia et al., 2019; A. Ornoy et al., 2015). Como ainda não foi estabelecido marcadores biológicos definitivos de autismo, o diagnóstico depende de uma série de sinais comportamentais. TEA é caracterizado como um transtorno no neurodesenvolvimento que promove deficiências na interação e comunicação social recíproca e interesses restritos, bem como comportamentos estereotipados repetitivos (Guo et al., 2019; Maia et al., 2019; Wang et al., 2017).

Durante as duas últimas décadas foi detectado um aumento crescente da prevalência do TEA. Centenas de estudos foram realizados, no entanto, ainda não foi esclarecido por que a incidência de autismo aumentou rapidamente durante os anos 90 e continua aumentando nos anos 2000. Os resultados das estimativas baseadas na população de autistas em março de 2014 de acordo com a Vigilância de Rede de Monitoramento e Desenvolvimento (ADDM) em várias comunidades dos EUA, como relatado pelos Centros de Controle e Prevenção de Doenças (CDC), indica uma prevalência geral de TEA de 14,7 por 1000 (IC $95 \%=14,3-5,1)$ ou uma em 68 crianças com 8 anos em 2010 (Guo et al., 2019).

Tanto a pesquisa genética quanto a ambiental resultaram no reconhecimento da complexidade etiológica do Transtorno do Espectro Autista (TEA). Um perfil metabólico integrado que reflete a interação de fatores genéticos, epigenéticos, ambientais e fatores endógenos que perturbam a via de interesse precisam ser avaliados (Iglesias Vázquez et al., 2018; A. Ornoy et al., 2015; Wang et al., 2017). Embora a comunidade científica tenha estabelecido que o TEA é uma condição multifatorial envolvendo genética e uma ampla gama de fatores de risco ambiental, somente na última década a pesquisa sobre fatores de risco ambiental cresceu significativamente (Gao et al., 2016; Virk et al., 2016; Yu et al., 2017).

Durante um longo período, foi considerada como baixa a contribuição de fatores ambientais, em parte devido à alta concordância monozigótica de gêmeos em estudos anteriores e em parte devido a uma limitada compreensão das interações gene-ambiente (Virk et al., 2016). Porém, nos últimos 10 anos, estudos com agentes biológicos como vias plausíveis, focadas em períodos críticos de desenvolvimento neurológico, resultaram em promessas de fatores de risco e proteção. Uma dessas áreas de pesquisa diz respeito a riscos nutricionais como fatores potencialmente modificáveis.

Apesar de vários estudos avaliarem a dieta e o estado nutricional em crianças afetadas com TEA, ainda existe escassez de pesquisas que investiguem diretamente a associação entre nutrição materna e risco de TEA na prole. A nutrição materna é essencial para o desenvolvimento do cérebro fetal, e deficiências nutricionais têm sido associadas a um aumento significativo do risco de vários efeitos adversos resultados de desenvolvimento neurológico, incluindo Defeitos do Tubo Neural e Transtorno do Espectro Autista (Altamimi, 2018; Schmidt et al., 2019; Sharman Moser et al., 2019; Zou et al., 2019). 
O cérebro fetal e seu desenvolvimento em termos de estrutura e função demonstrou ser influenciado pelo nutriente materno, devido ao aumento da demanda metabólica do feto em crescimento (DeVilbiss et al., 2017; Fluegge, 2018). O ácido fólico, por sua vez, a partir da ingestão de 400 microgramas por dia, demonstrou importância significativa para o desenvolvimento neuro-cognitivo durante a gestação (Fluegge, 2018). Portanto, é bastante plausível que o estado nutricional materno antes e durante a gravidez possa influenciar o risco de TEA.

Neste artigo, a associação entre ingestão materna de ácido fólico, incluindo suplementação de ácido fólico e o risco de TEA na prole será avaliado. Mais especificadamente, será investigado a administração de uma alta dosagem de ácido fólico durante o período periconcepcional e o desenvolvimento de TEA em descendentes. É relevante ressaltar que, embora existam evidências que a ingestão de folato durante a gravidez diminui o risco de TEA, pesquisas recentes especulam que uma alta ingestão materna de folato possa estar ligada a um aumento da prevalência de TEA, devido à fortificação de alimentos com ácido fólico e à uma suplementação indiscriminada de ácido fólico durante o período periconcepcional.

\section{Metodologia}

Esta revisão sistemática de literatura foi realizada por meio de uma síntese de evidências, interpretando criticamente as pesquisas de relevância disponíveis a respeito da administração da alta dose de ácido fólico no período periconcepcional e a sua influência no desenvolvimento do Transtorno do Espectro Autista.

O método escolhido foi o Prisma (Preferred Reporting Items for Systematic Reviews and Meta-Analyses), que consiste em uma revisão do Quorom (Quality Of Reporting Of Metaanalyses), que por sua vez, permite aprimorar a qualidade dos estudos de revisão sistemática.

Inicialmente, o presente estudo incluiu, todos os tipos de estudos encontrados sob a forma de artigo científico, datados de 2015 a 2020, nas bases de dados eletrônica: Lilacs, SciELO e PubMed. Restringiu-se à analise os artigos escritos nos idiomas inglês e português. Para a coleta de dados, os descritores utilizados foram: 'Folic Acid' AND 'Autism Espectrum Disorder'.

Os critérios de exclusão foram a abordagem de condutas terapêuticas, artigos de opiniões e comentários, amostra não significativa, informações repetidas ou disponíveis em outros artigos e artigos que não correspondiam com o propósito deste estudo. Diante disso, inicialmente o número de artigos encontrados foi de 56 e, após a adoção dos critérios de exclusão, o presente trabalho contemplou a inclusão de 34 artigos. A Figura 1 mostra a representação gráfica do processo de seleção dos artigos que foram utilizados nesta revisão. 
Figura 1 - Representação gráfica do processo de seleção dos artigos resultantes da busca.

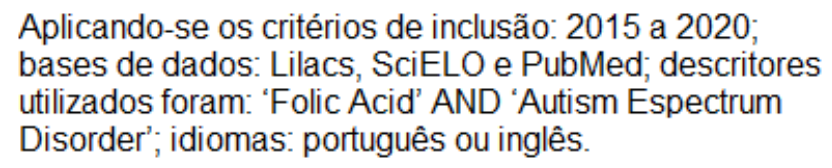

Aplicando-se os critérios de inclusão: 2015 a 2020; bases de dados: Lilacs, SciELO e PubMed; descritores utilizados foram: 'Folic Acid' AND 'Autism Espectrum Disorder'; idiomas: português ou inglês. TOTAL = 56 ARTIGOS

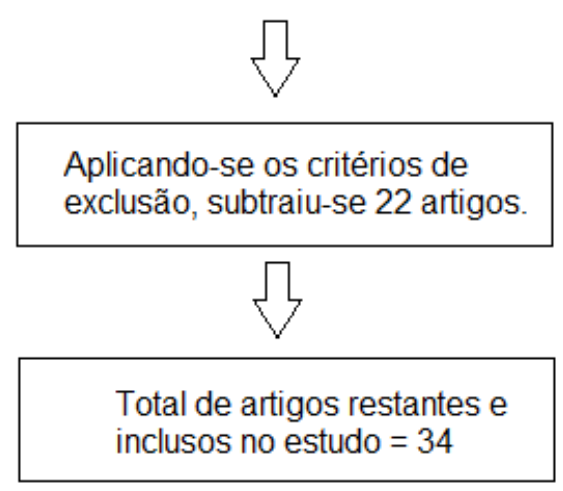

Fonte: Autores.

\section{Resultados e Discussão}

\subsection{Metabolismo do ácido fólico}

O folato ingerido pela dieta está, em sua maior parte, na forma de poliglutamatos reduzidos, ligados a proteínas. Para que ocorra a absorção, as formas reduzidas necessitam perder resíduos de glutamato. Esse processo ocorre a partir da enzima pteroilpoliglutamato hidrolase, que por sua vez, converte poliglutamatos reduzidos em formas oxidadas (Fluegge, 2018).

No lúmen do intestino delgado, os monoglutamatos formados após esse processo são absorvidos mediante mecanismo de transporte ativo, sendo o seu terço proximal o principal local de absorção. O processo de transporte ativo ocorre a partir de proteínas ligantes de folato presentes nas células do lúmen intestinal, visto que possuem alta afinidade pelo folato. Todavia, quando em altas doses, o mecanismo de escolha para absorção da vitamina é o da difusão passiva.

Para que ocorra a absorção da membrana basolateral para o sangue, é necessária a ação de enzimas presentes na superfície da borda em escova dos enterócitos, convertendo-os em suas formas ativas a partir da re-adição de novos resíduos de poliglutamatos e voltando à forma reduzida. Nesse processo, a vitamina B12 também é necessária na captação e na utilização adequadas de folato. A mucosa do duodeno e da parte superior do jejuno é rica em dihidrofolato redutase, enzima necessária para a metilação do folato reduzido e absorvido a partir da dieta.

No que concerne ao transporte pela circulação até os tecidos, sabe-se que ocorre sob a forma de 5-metil-tetrahidrofolato (5-metil-THF), conjugada principalmente com a albumina ou com proteínas de alta afinidade por folato, e são metabolizados principalmente no fígado.

Cerca de 10 a 30 nmol/L corresponde à taxa plasmática de folato, enquanto nos eritrócitos sua concentração é de 10 a 30 vezes maior (Iglesias Vázquez et al., 2018). O ciclo enterohepático e a alimentação dessa vitamina proporcionam um suprimento constante de tetrahidrofolato. O fígado, a medula óssea e a mucosa gastrointestinal constituem os principais alvos dessa vitamina, para isso, a enzima dihidrofolato redutase catalisa a redução da dihidrofolato (DHF) para sua forma ativa tetrahidrofolato (THF).

O tetrahidrofolato secretado através da bile é resultado da redução e da metilação do ácido pteroilglutâmico, processo realizado no fígado. Por conseguinte, a tetrahidrofolato após ser secretada na bile, é reabsorvida pelo intestino e em seguida 
liberada aos tecidos. Essa via metabólica é responsável por disponibilizar cerca de $200 \mathrm{mcg} / \mathrm{dia}$ de folato para o metabolismo tecidual (Gao et al., 2016; Iglesias Vázquez et al., 2018; Yu et al., 2017).

Nos tecidos periféricos, a 5-metil-THF chega ao interior das células via um sistema de transporte específico. Para isso, perde o grupamento metil por cedê-lo à homocisteína para a síntese de metionina. $\mathrm{O}$ folato fica retido dentro da célula a partir de reações de adição de resíduos de glutamato à THF resultante, pela folipoliglutamato sintase. O armazenamento do folato é feito sob a forma de poliglutamato e a partir daí, somente poderão sair da célula se retornarem à forma de derivados monoglutâmicos. Os estoques corporais variam de 5 a $10 \mathrm{mg}$, dos quais cerca de metade encontra-se no fígado (2,7 a 15,6 mcg/g de tecido) (Iglesias Vázquez et al., 2018).

Formas metabolicamente ativas e inativas são excretadas pela via urinária, fecal e pela bile. Pela urina, são eliminados cerca de 1 a 10 mcg/dia como formas de pteridinas e ácido benzoilglutâmico. Nas fezes, é excretado frações alimentares não absorvidas, e a secreção biliar e a síntese por bactérias intestinais (Gao et al., 2016; Yu et al., 2017).

\section{2 Ó́cido fólico na embriogênese e epigenética.}

O ciclo do folato facilita a síntese de ácidos nucleicos e é responsável pela transferência de grupos metil para DNA e proteínas, a partir da via folato-metionina (Asher Ornoy et al., 2018; Surman et al., 2019; Zhang et al., 2018). Grupos metil adicionados aos resíduos de citosina na região promotora CpGs no DNA genômico são centrais à regulação da expressão gênica (Zhang et al., 2018).

Zhang et al. (2018) verificaram que o folato é um componente essencial na síntese, metilação e reparo do DNA, e é crítico durante o neurodesenvolvimento. A metilação do DNA é um importante regulador epigenético da expressão gênica, crescimento e tecido diferenciação. Neste artigo, foi levantada a hipótese de que alteração da metilação do DNA (via disfuncional de folato-metionina) pode estar relacionada à etiologia do TEA (Zhang et al., 2018).

Especula-se que fatores genéticos e ambientais desempenham um papel no desenvolvimento de TEA (Leppert et al., 2019; Lintas, 2019). Estudos de família e gêmeos fornecem fortes evidências apoiando a contribuição da genética no desenvolvimento da doença (Lintas, 2019; Strøm et al., 2018). Estudos genéticos moleculares descobriram que o TEA é possivelmente causado por diversas variantes genéticas, como mutações genéticas, polimorfismos de nucleotídeo único (SNPs), anormalidades cromossômicas e variações no número de cópias (CNVs). Devido à alta heterogeneidade do TEA, variantes genéticas únicas são encontradas em apenas uma pequena proporção dos casos de TEA (Krsička et al., 2017; Modabbernia et al., 2017; Zhou et al., 2018). As interações entre predisposição genética e fatores ambientais têm sido propostas como os principais mecanismos na etiologia do TEA (Gogou \& Kolios, 2020; Shaik Mohammad et al., 2016).

O folato funciona como coenzima em diversas reações envolvendo transferência de carbonos (radicais metílicos), incluindo síntese de purina e timidilato, metabolismo de diversos aminoácidos (especialmente serina e homocisteína, em ação conjunta com a cobalamina e o ácido ascórbico), metilação de aminas biogênicas e síntese proteica da metionina (Gogou \& Kolios, 2020; Y.-M. Li et al., 2018; Tan et al., 2020). O 5-metil-THF atua como doador do grupo metil na conversão da homocisteína em metionina, em reação catalisada pela metionina sintase, enzima que também necessita da cobalamina para sua atividade. Em seguida, a metionina é metabolizada para a síntese de S-adenosilmetionina (SAM), o principal doador de grupo metil na maioria das reações bioquímicas. Fenômenos como: síntese de creatina, fosfatidilcolina, mielina, metilação do DNA e de neurotransmissores, necessitam da SAM como doador do grupo metil (Y.-M. Li et al., 2018).

Limitações e outras alterações da via desses nutrientes podem comprometer a metilação da citosina, portanto, alterando e debilitando a síntese de DNA. Polimorfismos genéticos foram identificados em inúmeras enzimas envolvidas na via metabólica do folato que, por sua vez, comprometem a síntese de DNA. Tal fato está associado à redução das concentrações plasmáticas de 
folato em humanos, ao aparecimento de frações não metabolizadas na circulação sanguínea e à hiperhomocisteínemia (Gogou \& Kolios, 2020; Y.-M. Li et al., 2018; Tan et al., 2020).

Shaik Mohammad et al. (2016) examinou a utilidade clínica dos polimorfismos genéticos envolvidos na via do folato na previsão do risco de distúrbios do espectro do autismo (TEA) e abordou as inconsistências na associação de MTHFR C677T e hiper-homocisteinemia com TEA. Neste estudo, foi desenvolvido um modelo de sistema de rede neural artificial (ANN) a partir dos dados de 138 crianças autistas e 138 não-autistas, usando os polimorfismos: GCPII C1561T, SHMT1 C1420T, MTHFR C677T, MTR A2756G e MTRR A66G, para explorar preditores de risco de autismo e determinantes genéticos da homocisteína. Meta-análises foram realizadas em 1361 crianças com TEA e 6591 crianças não-autistas para explorar a associação de MTHFR C677T e homocisteína com o risco de TEA (Shaik Mohammad et al., 2016).

Os resultados desta pesquisa identificaram que polimorfismos genéticos da via do folato foram preditores moderados de risco de autismo. MTHFR C677T e hiper-homocisteinemia foram identificados como fatores de risco para o autismo em todo o mundo. Interações sinérgicas entre MTHFR C677T e MTRR A66G aumentam a homocisteína.

O modelo de sistema de rede neural artificial mostrou 63,8\% de precisão na previsão do risco de autismo. Hiperhomocisteinemia foi observada em crianças autistas $(9,67 \pm 4,82$ vs. 6,99 $\pm 3,21 \mu \mathrm{mol} / 1)$. O sistema mostrou interações sinérgicas entre MTHFR C677T e MTRR A66G e como consequência aumentaram os níveis de homocisteína. Enquanto a metaanálise mostrou que o MTHFR é um fator de risco genético para o autismo em efeitos fixos (odds ratio: 1,47, intervalo de confiança de 95\%: 1,31-1,65) e efeitos aleatórios (odds ratio: 1,57, intervalo de confiança de 95\%: 1,16 -2.11) modelos. A metaanálise de nove estudos mostrou a hiper-homocisteinemia como um fator de risco significativo para o autismo nos modelos de efeitos fixos $(\mathrm{P}<0,0001)$ e efeitos aleatórios $(\mathrm{P}=0,026)$.

Lintas, (2019) encontrou evidências crescentes de que a regulação epigenética desempenha um papel importante no desenvolvimento neurológico. Nos seres humanos, o folato é um dos principais doadores do grupo metil necessário para a síntese de S-adenosilmetionina, que por sua vez é necessária para a metilação do DNA e da histona, importantes processos de neurodesenvolvimento. No presente artigo foi realizada uma revisão sistêmica que incluíram estudos de caso-controle. Foi encontrado, a partir dos resultados que a deficiência de folato durante a gravidez tem sido correlacionada com defeitos do tubo neural e com maior incidência de déficits neurocognitivos e/ou neurocomportamentais (Lintas, 2019).

\subsection{Relação entre o uso de ácido fólico e o desenvolvimento de tea}

No que concerne à etiologia do desenvolvimento do Transtorno do Espectro Autista (TEA), há inúmeras divergências e questões a serem respondidas pelo meio científico. Sabe-se que o folato é necessário para processos metabólicos e desenvolvimento neural (Cheng et al., 2019; M. Li et al., 2019). Por causa disso, garantir seus níveis adequados para mulheres grávidas através da suplementação com ácido fólico sintético (FA), a fim de evitar defeitos no tubo neural, tem sido uma iniciativa de saúde pública em andamento (Levine et al., 2018; Wang et al., 2017).

No entanto, a suplementação em alta dosagem com multivitaminas contendo ácido fólico sintético antes e durante a gravidez, concomitantemente com folatos alimentares naturais levou a uma demografia com níveis séricos altos. Isso levanta preocupações sobre os efeitos prejudiciais dos altos níveis plasmáticos de ácido fólico sintético, incluindo um aumento no risco de transtorno do espectro do autismo (TEA) (Raghavan et al., 2018; Wiens \& DeSoto, 2017). Uma linha de pesquisa recente examina a relação da suplementação de uma alta dose de ácido fólico antes e durante a gravidez com o desenvolvimento do TEA.

Tal fato ocorre, desde que os EUA, a partir de $1^{\circ}$ de janeiro de 1998 e por estabelecimento da Food and Drug Administration (FDA), promoveu a fortificação obrigatória de produtos manufaturados de cereais. Desde então, houve uma diminuição significativa nos casos de defeitos do tubo neural (DTN) nos EUA (Guo et al., 2019; A. Ornoy et al., 2015; Wang et 
al., 2017) . No entanto, vários pesquisadores, a partir de estudos epidemiológicos questionaram se um aumento do folato materno no período periconcepcional poderia estar parcialmente relacionado ao aumento inexplicável de casos de TEA nos EUA ocorridos na mesma época (Wiens \& DeSoto, 2017). É relevante ressaltar que o fechamento do tubo neural e, portanto, sua ativação pela suplementação com ácido fólico, ocorre em um momento durante a embriogênese que também é crítica no desenvolvimento do autismo.

Está bem estabelecido que houve um aumento significativo do status materno do folato desde que a FDA determinou a fortificação de certos alimentos (1998), que resultaram em menor incidência de DTN em meados dos anos 2000 e um aumento na prevalência de autismo e distúrbios relacionados nos EUA. Os investigadores se perguntam se esses prazos semelhantes para a mudança no status do folato materno e possível prevalência de autismo são eventos aleatórios ou então, de fato, houveram um papel na prevalência de TEA (Raghavan et al., 2018; Wiens \& DeSoto, 2017).

Para explorar possíveis associações entre o uso materno de suplementos de micronutrientes e os níveis e sintomas subseqüentes de vitaminas na prole com transtorno do espectro do autismo (TEA), Tan et al. (2020) selecionou um total de 416 crianças com TEA e 201 crianças com desenvolvimento típico (TD) para inclusão do seu estudo. Foram dosados os níveis de ácido fólico em recém nascidos, durante o período do puerpério, e foi concluído que as crianças nascidas de mães sem ácido fólico (FA) durante a gravidez apresentaram comprometimentos cognitivos sociais mais graves, comprometimentos da comunicação social, maneirismos do comportamento do autismo, atrasos no desenvolvimento do comportamento motor bruto e adaptativo e problemas gastrointestinais do que os filhos de mães que usaram o FA $(\mathrm{P}<0,05)$. Houve uma associação entre a suplementação materna de micronutrientes e níveis menores de folato nas crianças com TEA $(\mathrm{P}<0,05)$.

Li et al. (2019) descreveu que a nutrição pré-natal é um fator crítico para o desenvolvimento do cérebro fetal. O autor revisou sistematicamente a associação entre nutrição pré-natal com o risco de descendentes com distúrbios do desenvolvimento neurológico. Os resultados do desenvolvimento neurológico incluíram distúrbios do espectro do autismo (TEA), déficit de atenção e hiperatividade (TDAH) e deficiências intelectuais. Como resultado da pesquisa, o autor encontrou uma associação inversa geral entre a suplementação com ácido fólico materno ou multivitamínico e o risco de TEA em crianças.

Cheng et al. (2019) identificaram que o folato, ômega-3, vitamina D3, prevenção de toxinas ambientais, correção de deficiências, reforço imunológico e amamentação prolongada estão todos relacionados à possível redução de resultados adversos na gravidez, incluindo TEA. Resultados semelhantes foram encontrados por Iglesias Vázquez et al.(2018), em uma revisão sistemática que avaliou que os suplementos pré-natais de rotina de ácido fólico estavam associados a níveis significativamente mais baixos de TEA, apesar disso, ressaltou que os resultados devem ser interpretados com cautela.

Em um estudo de caso-controle para registrar crianças em desenvolvimento típico e crianças com TEA, Li et al. (2018) coletou dados derivados do Autism Clinical and Environmental Database (ACED). Foram incluídas trezentos e setenta e quatro crianças com autismo e 354 crianças com desenvolvimento típico. Essa pesquisa revelou que os padrões alimentares desequilibrados maternos antes da concepção tinham um aumento significativo do risco de TEA na prole.

Zou et al. (2019) verificou que os distúrbios do espectro do autismo (TEA) são uma prevalência crescente de distúrbios do neurodesenvolvimento, mas faltam biomarcadores confiáveis para o diagnóstico precoce. O presente estudo investigou 13 sorologias de metabólitos e duas variantes genéticas relacionadas ao metabolismo do folato em um total de 89 casos de TEA e 89 controles correspondentes. Dez metabolitos foram significativamente diferentes entre os grupos, dos quais seis metabólitos foram utilizados como preditores para determinar a previsão discriminante modelo: vitamina B12, 5-metileno-tetra-hidrofolato, metonina, a proporção de S-adenosilmetionina / S-adenosil-homocisteína, metionina sintase e transcobalamina II. O modelo teve significância estatística (lambda $=0,520,2=113,103, \mathrm{df}=6, \mathrm{P}<0,001$ ) e identificou corretamente 84,3\% dos grupos de TEA e normais. Com a referida pesquisa, consegue-se maiores esclarecimentos no diagnóstico precoce de TEA a partir da identificação de níveis plásmaticos menores dos biomarcadores. 
Levine et al. (2018) examinaram as associações entre o uso de ácido fólico e suplementos multivitamínicos antes e durante a gestação com o risco de TEA, a partir de um estudo de coorte de caso-controle de 45300 crianças israelenses nascidas entre 1 de janeiro de 2003 e 31 de dezembro de 2007. Foi acompanhado desde o nascimento até 26 de janeiro de 2015 para a avaliação de sinais clínicos concordantes de TEA. Os casos foram todas crianças diagnosticadas com TEA e os controles foram uma amostra aleatória de $33 \%$ de todas as crianças nascidas vivas. Identificou-se que a exposição materna ao ácido fólico e/ou suplementos multivitamínicos durante a gravidez foi estatisticamente significativamente associada a uma menor probabilidade de TEA na prole em comparação com a não exposição durante a gravidez (RR, 0,27; IC 95\%, 0,22-0,33; P<0,001).

Nas últimas duas décadas foi amplamente disseminado o uso de suplementação de ácido fólico em multivitamínicos. Por outro lado, altos níveis de suplementação de FA durante a gravidez podem não ser necessários e não são isentos de riscos. O papel dos folatos na síntese de nucleotídeos e nas reações de metilação como doador de metila é fundamental para praticamente todos os aspectos do desenvolvimento e da saúde. No entanto, níveis inesperadamente altos de FA podem ter implicações inadvertidas para a metilação adequada do DNA durante tempos de rápida divisão celular, como no desenvolvimento pré-natal.

Uma alta dose de ácido fólico administrada durante a gestação sem um direcionamento clínico e seus efeitos não devem ser subestimados. Wiens \& DeSoto (2017) relatou os motivos pelos quais a suplementação excessiva de AG é uma preocupação. O estudo examinou os efeitos da FA no desenvolvimento neuronal a partir do estudo de experimentos de cultura de tecidos. Com base nas evidências avaliadas, o estudo concluiu que é necessário cuidado com o excesso de suplementação.

Em uma das pesquisas revisadas na revisão sistemática de Wiens \& DeSoto (2017), foi examinado áreas de gânglios da raiz dorsal de embriões de galos (DRG). Em seguida, comparou o comportamento de cones de crescimento e neurites antes e depois da adição de FA. Examinaram-se culturas de amostras, a partir da imunohistoquímica, para avaliar as redes neurais, vesículas sinápticas e para enumerar e medir comprimentos de neurites.

Eles descobriram que a quantidade de neurotransmissores emitida pelos neurônios não foi afetada. Observou-se ainda, uma relação dependente entre o comprimento das neurites e a concentração de FA: uma inibição significativa da extensão das neurites. O comprimento médio foi menor. No entanto, eles relataram que o total médio de áreas sinaptogênicas coradas ao redor de cada DRG cultivado foi reduzido significativamente. Por fim, eles acumularam dados sobre uma diminuição do comportamento dinâmico de área dos cones de crescimento. Embora este estudo tenha usado concentrações de AF que eram (como nos estudos com roedores) bastante altas e não deviam ser extrapoladas para os níveis observados em seres humanos, os resultados confirmam a idéia de um efeito direto teórico.

Os resultados desta pesquisa sugerem que a FA pode estar envolvida em uma competição direta pela ligação ao importante receptor N-metil-d-aspartato (NMDA) na sinapse. Esse receptor é conhecido por ser importante não apenas na membrana pós-sináptica, mas também na membrana pré-sináptica nos cones de crescimento durante o processo de embriogênese. A estrutura química da FA contém glutamato em uma extremidade, o abundante neurotransmissor envolvido na abertura do canal receptor NMDA para fluxos de íons. Alterar a concentração de íons cálcio nos neurônios teria efeitos profundos no desenvolvimento neural, porque a concentração citoplasmática local de íons cálcio é um regulador chave da extensão dos neurites, formação de sinapses e maturação da coluna sináptica e dendrítica. Em suma, são fortes as evidências de que o aumento da FA além das quantidades recomendadas não está isento de riscos.

Raghavan et al. (2018) realizou uma análise prospectiva entre a associação de suplementação multivitamínica durante a gravidez e medidas dos níveis plasmáticos de biomarcadores de folato materno e vitamina B12 no nascimento e risco de Transtorno do Espectro do Autismo (TEA) da criança. Esta pesquisa incluiu 1257 pares mãe-filho, que foram recrutados no nascimento e acompanhados prospectivamente durante a infância no Boston Medical Center. TEA foi definido a partir de códigos de diagnóstico em registros médicos eletrônicos e a suplementação multivitamínica materna foi avaliada por meio de entrevista por questionário, além disso, o folato plasmático materno e a vitamina B12 foram medidos a partir de amostras colhidas 2-3 dias 
após o nascimento. Verificou-se que a suplementação moderada autorreferida (3-5 vezes/semana) durante a gravidez foi associada à diminuição do risco de TEA, achado consistente com a maior parte de pesquisas anteriores. No entanto, a suplementação baixa ( $\leq 2$ vezes/semana) e alta ( $>5$ vezes/semana) foi associada ao aumento do risco de TEA. Níveis muito altos de folato plasmático materno ao nascimento ( $\geq 60,3 \mathrm{nmol} / \mathrm{L}$ ) tiveram risco 2,5 vezes maior de TEA (intervalo de confiança de $95 \%[\mathrm{IC}])$.

Níveis extremamente elevados de folato no plasma materno e de B12 ao nascimento foram associados ao risco de TEA. Este estudo gerador de hipóteses não questiona a importância de consumir ácido fólico e vitamina B12 adequados durante a gravidez, mas levanta novas questões sobre o impacto de níveis extremamente elevados de folato plasmático e sobre a exposição à vitamina B12 no útero no desenvolvimento inicial do cérebro.

\section{Conclusão}

Esta revisão conclui que são necessárias pesquisas adicionais com desenhos de estudos adequadamente padronizados e com metodologia adequada antes que quaisquer conclusões definitivas possam ser tomadas. Nenhum estudo que avalie o fator protetivo de ácido fólico em altas dosagens para TEA foi encontrado nesta revisão sistemática e dois estudos demonstraram efeitos negativos para o neurodesenvolvimento, a partir de ensaios clínicos com animais e pesquisas in vitro. Mais pesquisas devem ser realizadas na tentativa de comprovar se o tempo e a quantidade do uso dessa vitamina podem estar implicados no desenvolvimento do TEA.

O transtorno do Espectro Autista e a sua etiologia, bem como sua fisiopatologia, ainda não é completamente esclarecida. Linhas de pesquisas recentes, de forma crescente, buscam comprovar que a suplementação em alta dosagem de ácido fólico é um dos elementos que contribuem com o desenvolvimento do TEA. Os resultados de estudos clínicos recentes, apesar de poucos e inconclusivos, levantam questões sobre dosagens adequadas ou uso mais direcionado do ácido fólico que necessitam ser investigadas. Tais linhas de pesquisa seriam interessantes como sugestões de pesquisa para trabalhos futuros.

\section{Referências}

Altamimi, M. (2018). Could Autism Be Associated With Nutritional Status in the Palestinian population? The Outcomes of the Palestinian Micronutrient Survey. Nutrition and Metabolic Insights, 11, 117863881877307. https://doi.org/10.1177/1178638818773078

Cheng, J., Eskenazi, B., Widjaja, F., Cordero, J. F., \& Hendren, R. L. (2019). Improving autism perinatal risk factors: A systematic review. Medical Hypotheses, 127, 26-33. https://doi.org/10.1016/j.mehy.2019.03.012

DeVilbiss, E. A., Magnusson, C., Gardner, R. M., Rai, D., Newschaffer, C. J., Lyall, K., Dalman, C., \& Lee, B. K. (2017). Antenatal nutritional supplementation and autism spectrum disorders in the Stockholm youth cohort: population based cohort study. BMJ, j4273. https://doi.org/10.1136/bmj.j4273

Fluegge, K. (2018). Re: Folate status and autism spectrum disorders. Paediatric and Perinatal Epidemiology, 32(3), 308-308. https://doi.org/10.1111/ppe.12449

Gao, Y., Sheng, C., Xie, R., Sun, W., Asztalos, E., Moddemann, D., Zwaigenbaum, L., Walker, M., \& Wen, S. W. (2016). New Perspective on Impact of Folic Acid Supplementation during Pregnancy on Neurodevelopment/Autism in the Offspring Children - A Systematic Review. PLOS ONE, 11(11), e0165626. https://doi.org/10.1371/journal.pone.0165626

Gogou, M., \& Kolios, G. (2020). Nutritional Supplements During Gestation and Autism Spectrum Disorder: What Do We Really Know and How Far Have We Gone? Journal of the American College of Nutrition, 39(3), 261-271. https://doi.org/10.1080/07315724.2019.1635920

Guo, B.-Q., Li, H.-B., Zhai, D.-S., \& Ding, S.-B. (2019). Association of maternal prenatal folic acid intake with subsequent risk of autism spectrum disorder in children: A systematic review and meta-analysis. Progress in Neuro-Psychopharmacology and Biological Psychiatry, 94, 109650. https://doi.org/10.1016/j.pnpbp.2019.109650

Iglesias Vázquez, L., Canals, J., \& Arija, V. (2018). Review and meta-analysis found that prenatal folic acid was associated with a 58\% reduction in autism but had no effect on mental and motor development. Acta Paediatrica, apa.14657. https://doi.org/10.1111/apa.14657

Krsička, D., Geryk, J., Vlčková, M., Havlovicová, M., Macek, M., \& Pourová, R. (2017). Identification of likely associations between cerebral folate deficiency and complex genetic- and metabolic pathogenesis of autism spectrum disorders by utilization of a pilot interaction modeling approach. Autism Research, 10(8), 1424-1435. https://doi.org/10.1002/aur.1780

Leppert, B., Havdahl, A., Riglin, L., Jones, H. J., Zheng, J., Davey Smith, G., Tilling, K., Thapar, A., Reichborn-Kjennerud, T., \& Stergiakouli, E. (2019). Association of Maternal Neurodevelopmental Risk Alleles With Early-Life Exposures. JAMA Psychiatry, 76(8), 834. 
https://doi.org/10.1001/jamapsychiatry.2019.0774

Levine, S. Z., Kodesh, A., Viktorin, A., Smith, L., Uher, R., Reichenberg, A., \& Sandin, S. (2018). Association of Maternal Use of Folic Acid and Multivitamin Supplements in the Periods Before and During Pregnancy With the Risk of Autism Spectrum Disorder in Offspring. JAMA Psychiatry, $75(2), 176$. https://doi.org/10.1001/jamapsychiatry.2017.4050

Li, M., Francis, E., Hinkle, S. N., Ajjarapu, A. S., \& Zhang, C. (2019). Preconception and Prenatal Nutrition and Neurodevelopmental Disorders: A Systematic Review and Meta-Analysis. Nutrients, 11(7), 1628. https://doi.org/10.3390/nu11071628

Li, Y.-M., Shen, Y.-D., Li, Y.-J., Xun, G.-L., Liu, H., Wu, R.-R., Xia, K., Zhao, J.-P., \& Ou, J.-J. (2018). Maternal dietary patterns, supplements intake and autism spectrum disorders. Medicine, 97(52), e13902. https://doi.org/10.1097/MD.0000000000013902

Lintas, C. (2019). Linking genetics to epigenetics: The role of folate and folate-related pathways in neurodevelopmental disorders. Clinical Genetics, 95(2), 241-252. https://doi.org/10.1111/cge.13421

Maia, C. S., Menezes, K. M. C., Tenorio, F. C. A. M., Queiroz Júnior, J. R. A., \& Maciel, G. E. S. (2019). Transtorno do espectro autista e a suplementação por ácido fólico antes e durante a gestação. Jornal Brasileiro de Psiquiatria, 68(4), 231-243. https://doi.org/10.1590/0047-2085000000251

Modabbernia, A., Velthorst, E., \& Reichenberg, A. (2017). Environmental risk factors for autism: an evidence-based review of systematic reviews and metaanalyses. Molecular Autism, 8(1), 13. https://doi.org/10.1186/s13229-017-0121-4

Ornoy, A., Weinstein-Fudim, L., \& Ergaz, Z. (2015). Prenatal factors associated with autism spectrum disorder (ASD). Reproductive Toxicology, 56, 155-169. https://doi.org/10.1016/j.reprotox.2015.05.007

Ornoy, Asher, Koren, G., \& Yanai, J. (2018). Is post exposure prevention of teratogenic damage possible: Studies on diabetes, valproic acid, alcohol and anti folates in pregnancy: Animal studies with reflection to human. Reproductive Toxicology, 80, 92-104. https://doi.org/10.1016/j.reprotox.2018.05.006

Raghavan, R., Riley, A. W., Volk, H., Caruso, D., Hironaka, L., Sices, L., Hong, X., Wang, G., Ji, Y., Brucato, M., Wahl, A., Stivers, T., Pearson, C., Zuckerman, B., Stuart, E. A., Landa, R., Fallin, M. D., \& Wang, X. (2018). Maternal Multivitamin Intake, Plasma Folate and Vitamin B 12 Levels and Autism Spectrum Disorder Risk in Offspring. Paediatric and Perinatal Epidemiology, 32(1), 100-111. https://doi.org/10.1111/ppe.12414

Schmidt, R. J., Iosif, A.-M., Guerrero Angel, E., \& Ozonoff, S. (2019). Association of Maternal Prenatal Vitamin Use With Risk for Autism Spectrum Disorder Recurrence in Young Siblings. JAMA Psychiatry, 76(4), 391. https://doi.org/10.1001/jamapsychiatry.2018.3901

Shaik Mohammad, N., Sai Shruti, P., Bharathi, V., Krishna Prasad, C., Hussain, T., Alrokayan, S. A., Naik, U., \& Devi, A. R. R. (2016). Clinical utility of folate pathway genetic polymorphisms in the diagnosis of autism spectrum disorders. Psychiatric Genetics, 26(6), 281-286. https://doi.org/10.1097/YPG.0000000000000152

Sharman Moser, S., Davidovitch, M., Rotem, R. S., Chodick, G., Shalev, V., \& Koren, G. (2019). High dose folic acid during pregnancy and the risk of autism; The birth order bias: A nested case-control study. Reproductive Toxicology, 89, 173-177. https://doi.org/10.1016/j.reprotox.2019.07.083

Strøm, M., Granström, C., Lyall, K., Ascherio, A., \& Olsen, S. F. (2018). Research Letter: Folic acid supplementation and intake of folate in pregnancy in relation to offspring risk of autism spectrum disorder. Psychological Medicine, 48(6), 1048-1054. https://doi.org/10.1017/S0033291717002410

Surman, C., Ceranoglu, A., Vaudreuil, C., Albright, B., Uchida, M., Yule, A., Spencer, A., Boland, H., Grossman, R., Rhodewalt, L., Fitzgerald, M., \& Biederman, J. (2019). Does 1-Methylfolate Supplement Methylphenidate Pharmacotherapy in Attention-Deficit/Hyperactivity Disorder? Journal of Clinical Psychopharmacology, 39(1), 28-38. https://doi.org/10.1097/JCP.0000000000000990

Tan, M., Yang, T., Zhu, J., Li, Q., Lai, X., Li, Y., Tang, T., Chen, J., \& Li, T. (2020). Maternal folic acid and micronutrient supplementation is associated with vitamin levels and symptoms in children with autism spectrum disorders. Reproductive Toxicology, 91, 109-115. https://doi.org/10.1016/j.reprotox.2019.11.009

Virk, J., Liew, Z., Olsen, J., Nohr, E. A., Catov, J. M., \& Ritz, B. (2016). Preconceptional and prenatal supplementary folic acid and multivitamin intake and autism spectrum disorders. Autism, 20(6), 710-718. https://doi.org/10.1177/1362361315604076

Wang, M., Li, K., Zhao, D., \& Li, L. (2017). The association between maternal use of folic acid supplements during pregnancy and risk of autism spectrum disorders in children: a meta-analysis. Molecular Autism, 8(1), 51. https://doi.org/10.1186/s13229-017-0170-8

Wiens, D., \& DeSoto, M. (2017). Is High Folic Acid Intake a Risk Factor for Autism?-A Review. Brain Sciences, 7(12), 149. https://doi.org/10.3390/brainsci7110149

Yu, X.-F., Li, M., \& Zheng, Y. (2017). [Association between maternal folate supplementation during pregnancy and the risk of autism spectrum disorder in the offspring: a Meta analysis]. Zhongguo Dang Dai $\mathrm{Er} \mathrm{Ke} \mathrm{Za}$ Zhi $=$ Chinese Journal of Contemporary Pediatrics, 19(3), $286-291$. https://doi.org/10.7499/j.issn.1008-8830.2017.03.007

Zhang, Z., Yu, L., Li, S., \& Liu, J. (2018). Association Study of Polymorphisms in Genes Relevant to Vitamin B12 and Folate Metabolism with Childhood Autism Spectrum Disorder in a Han Chinese Population. Medical Science Monitor, 24, 370-376. https://doi.org/10.12659/MSM.905567

Zhou, J., Liu, A., He, F., Jin, Y., Zhou, S., Xu, R., Guo, H., Zhou, W., Wei, Q., \& Wang, M. (2018). High prevalence of serum folate receptor autoantibodies in children with autism spectrum disorders. Biomarkers, 23(7), 622-624. https://doi.org/10.1080/1354750X.2018.1458152

Zou, M., Sun, C., Liang, S., Sun, Y., Li, D., Li, L., Fan, L., Wu, L., \& Xia, W. (2019). Fisher discriminant analysis for classification of autism spectrum disorders based on folate-related metabolism markers. The Journal of Nutritional Biochemistry, 64, 25-31. https://doi.org/10.1016/j.jnutbio.2018.09.023 